\title{
Genetic and Preventive Services for Hereditary Breast and Ovarian Cancer in the Czech Republic
}

\author{
Lenka Foretova', Katarina Petrakova', Marketa Palacova', Renata Kalabova', Marie Navratilova', Miroslava Lukesova', \\ Petra Vasickova', Eva Machackova', Zdenek Kleibl', Petr Pohlreich²
}

${ }^{1}$ Masaryk Memorial Cancer Institute Brno; ${ }^{2}$ Department of Biochemistry and Experimental Oncology, First Faculty of Medicine, Charles University Prague

Key words: hereditary, breast/ovarian cancer

Corresponding author: Lenka Foretova, Department of Cancer Epidemiology and Genetics, Masaryk Memorial Cancer Institute, Zluty kopec 7, 65653 Brno, Czech Republic; phone and fax: +4205 4313 6907; e-mail: foretova@mou.cz

Submitted: 2 October 2005

Accepted: 15 November 2005

\begin{abstract}
The majority of hereditary breast and ovarian cancers can be accounted for by germline mutations in the BRCA1 and BRCA2 genes. Genetic counselling and testing in high-risk patients in the Czech Republic began in 1997 in two centres (Masaryk Memorial Cancer Institute in Brno, $\mathrm{MMCl}$, and the General University Hospital plus the First Faculty of Medicine, Charles University in Prague, 1FMUK). Health insurance covers testing in $\mathrm{MMCl}$, whereas testing at 1 FMUK is covered by research grants. The spectrum of mutations in the BRCA1 gene is similar in the Bohemian (western) and Moravian (eastern) regions of the country but the mutation spectrum observed in the BRCA2 gene is completely different. There are three BRCA1 gene mutations that are responsible for $69 \%$ and $70.4 \%$ of all BRCA 1 mutations identified in women reporting to the Brno and Prague centres, respectively. The two most frequent mutations in the BRCA2 gene, which comprises $41.5 \%$ of all detected BRCA2 mutations in Brno, were not found in women tested in the Prague centre. The testing of BRCA1/BRCA2 or other possible predisposition genes for hereditary breast/ovarian cancer is determined by medical geneticists after genetic counselling. Predictive testing is offered to persons older than 18 years of age. Genetic counselling centres are easily accessible to all inhabitants in the country. Specialized preventive care is mostly organized by $\mathrm{MMCl}$ and the General University Hospital in Prague; however, some patients and their family members are under the care of other oncology departments and clinics. The quality of preventive care in different hospitals is currently being investigated.
\end{abstract}

\section{Introduction}

The incidence of breast cancer has doubled over the past twenty years in the Czech Republic. In 5-10\% of breast cancers, hereditary factors may be a cause of young onset, bilateral breast or ovarian cancer and familial accumulation of the disease. Genetic testing of BRCA1 and BRCA2 predisposition genes has been available in the Czech Republic since 1997 in two major molecular genetic laboratories - one in Brno (Masaryk Memorial Cancer Institute, $\mathrm{MMCl}$ ) and the other in Prague (Department of Biochemistry and Experimental Oncology, First Faculty of Medicine, Charles University).

Genetic counselling services for hereditary cancer were started at $\mathrm{MMCl}$ in Brno in 1997. Currently the department comprises a genetic clinic and a molecular genetic laboratory. The laboratory provides testing of BRCA1 and BRCA2 genes for about $65 \%$ of high- 


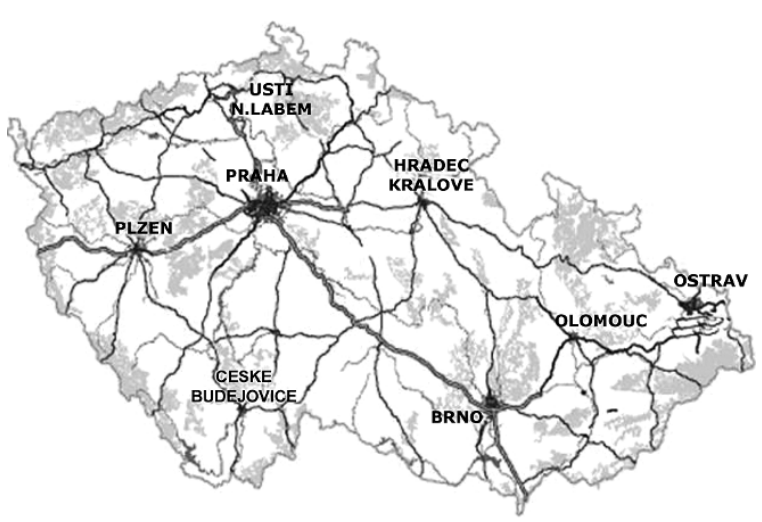

Fig. 1. The network of genetic centers offering oncology counselling in the Czech Republic

-risk patients tested in the Czech Republic. Counselling and genetic testing is covered by health insurance and the laboratory is accredited within the institute and undergoes EMQN quality testing annually. Eighty percent of tested patients are from the Moravian region (eastern) of the Czech Republic and 20\% from the Bohemian (western) region. The second major laboratory in Prague is part of the First Faculty of Medicine in Charles University. The testing in this setting is covered by grants and the laboratory undergoes EMQN quality assessment every year. All patients tested are from the Bohemian region (mostly from in and around Prague) of the Czech Republic. Recently, molecular genetic testing of BRCA1 and BRCA2 has begun in two private laboratories where individual cases are analysed.

Genetic counselling of high-risk patients or their families is now offered by medical geneticists working in 10 public genetic centres and in several private centres (Fig. 1). For persons wishing to be tested a counselling session prior to testing is provided and each patient who proceeds with testing must sign an informed consent document. Each patient receives a second counselling session at the time of being told their results and receives a very detailed genetic report.

\section{Methods of genetic testing}

The inclusion criteria for BRCA1 and BRCA2 testing are similar in both the Brno and Prague centres: i) families with three or more cases of breast or ovarian cancer diagnosed at any age; ii) families with only two cases of breast cancer, one at the age $<50$ years, with ovarian cancer at any age; iii) sporadic unilateral breast cancer $<40$ years (in Prague $<35$ years of age), sporadic unilateral ovarian cancer $<40$ years, breast and ovarian cancer at any age, male breast cancer iv) sporadic bilateral breast or ovarian cancer $<50$ years of age. In most families the testing is performed using DNA from a proband with breast or ovarian cancer having the highest probability of hereditary disease. In a small proportion of cases where no patient is living in a family the testing is performed on DNA from a high-risk healthy individual.

Genetic testing is based on genomic DNA in Brno and the analysis of DNA and RNA in the Prague laboratory. Mutation detection methods have been described previously by Foretova et al., 2004, and Pohlreich et al., 2005 [1, 2]. All positive results from the screening tests for BRCA1 and BRCA2 were analysed by automated sequencing and were confirmed on a second sample of independently isolated DNA. All positive and negative predictive testing results are also confirmed on a second sample.

\section{Testing results}

The spectrum of mutations found in the BRCA1 gene is similar in the Brno and Prague centres. The three most frequent mutations in the BRCA1 gene, which are responsible for about $69 \%$ of BRCA1 positive cases in Brno and $70.4 \%$ in Prague, are c.5385dupC, c.300T $>$ G and c.3819 3823delGTAAA according to the BIC information database (http://research.nhgri.nih.gov/bic/). Other BRCA1 mutations were found to be scattered throughout the whole coding region of the gene. Three novel mutations were detected in the BRCA1 gene in patients from Brno (c.2607_2616dup10, c.2881delA and c.3761_3762delGA) and one in Prague (c. 1866A>T) [1-3].

The results of BRCA2 testing show significant differences between the two centres. In Brno, two mutations, c.8138_8142delCCTTT and c.8765_8766delAG, were detected in $41.5 \%$ of all BRCA2 positive cases. These two mutations were not observed in patients from the Prague region. In Brno individual mutations in the BRCA2 gene were scattered between exons 3 and 25 (most were frame-shift or nonsense changes) whereas all mutations detected in patients in the Prague laboratory were located in exon 10 and 11 . Five novel mutations in BRCA2 were detected in Brno (c.5073_5074delCT, c.6677 6678deIAA, c.6866delC, c.6982dupT, c.8397_8400dupTGGG) and two in Prague (c.4167delC, c.5991 dupT) [1-3]. The spectrum of the five most frequent mutations in both genes is shown in fig. 2.

The mutation detection rate was similar in both centres. Overall mutations were discovered in $28.9 \%$ of all tested index cases from Brno and in 26.9\% from Prague. Thus far 700 index cases have been tested for BRCA1 and BRCA2 mutations in Brno and 245 patients in Prague. 


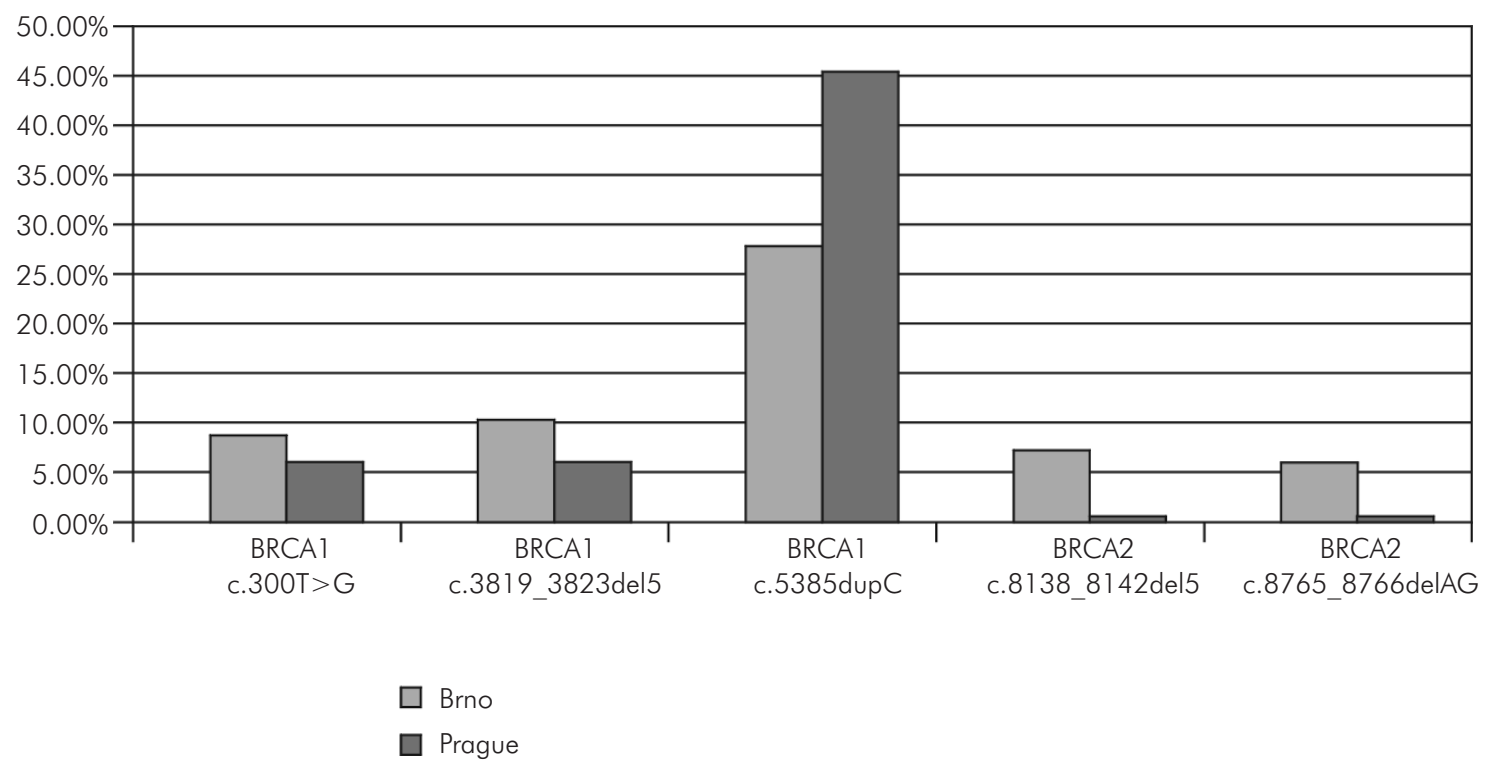

Fig. 2. Frequencies of the five most frequent mutations in BRCA1 and BRCA2 genes detected in Brno and Prague centres (\% of all detected mutations)

\section{Predictive testing}

Predictive testing is offered to relatives at risk and includes all men and women older than 18 years of age. All relatives have a genetic counselling session before and after testing. Predictive testing was offered to 445 relatives in Brno and 120 relatives in Prague.

\section{Preventive care}

Specialized preventive care is organized by both centres, within the Complex Oncology Treatment Clinic of $\mathrm{MMCl}$ in Brno, and the Department of Oncology of the General Faculty Hospital in Prague. Oncologists, gynaecologists and geneticists from both centres together with specialists from other hospitals came together to draw up and publish guidelines for the specialized preventive care of BRCA1 and BRCA2 carriers in the Czech journal Clinical Oncology 1/2003 [4]. In Brno, two specialized oncologists together with a gynaecologist and a gastroenterologist regularly see most of the BRCA1 and BRCA2 mutation carriers. Only a very small proportion of carriers (those treated by other oncologists or radiotherapists) are seen outside of the high-risk clinic. One specialized nurse, who is part of the genetics department, organizes all visits for carriers and other high-risk individuals. She coordinates appointments so that all planned examinations can be scheduled in one day. She invites patients by letter and phone for every visit. A similar organizational approach is used at the Prague General Faculty Hospital. Additional carriers who cannot be seen in Brno or Prague are clinically followed in other hospitals all over the country. A study comparing the quality of preventive care in different hospitals in the Czech Republic is currently under evaluation.

Prophylactic adnexectomy is offered to BRCAl and BRCA2 carriers as efficient primary prevention especially in families with hereditary breast and ovarian cancer. The role of preventive surgery is explained by both geneticists and gynaecologists. If a woman is adamant against having adnexectomy and repeatedly refuses to accept this strategy to reduce her risk of disease by the age of 40 years, she and her doctor sign a declaration of refusal for the protection of the gynaecologist.

Prophylactic mastectomies and breast reconstruction are explained to patients and offered to carriers as the most efficient way of primary breast cancer prevention. Women have access to a specialized plastic surgeon for consultation prior to the procedure, during which possible methods of reconstruction are discussed. The use of prophylactic mastectomies for the primary prevention of breast cancer is slowly increasing among highly motivated carriers. Prophylactic surgery to reduce cancer risk is covered by health insurance.

\section{Discussion}

Genetic counselling and testing for hereditary breast and/or ovarian cancer is available to all women in 
the Czech Republic who are at risk of developing early onset disease. There is access to genetic counselling in all regions of the country and two major molecular genetic laboratories provide BRCA1 and BRCA2 testing for eligible women. To ensure appropriate testing standards both laboratories undergo an annual quality assurance programme orchestrated by EMQN.

The three most frequent mutations in the BRCAl gene (c.5385dupC, c.300T>G and c.3819_3823delGTAAA) are seen at very similar frequencies in the Prague region and other parts of the Czech Republic. The three mutations are very frequently found in Europe and none of our carriers of the c.5385dupC change reported an Ashkenazi Jewish history. The mutation c. 4153 delA, which accounts for $9.8 \%$ of all BRCA 1 mutations identified in Poland, was not observed in patients tested from either Prague or Brno.

The spectrum of BRCA2 mutations was different in the Moravian and Bohemian (Prague) regions of the country. In the Brno series of patients, around $80 \%$ were living in the Moravian and $20 \%$ in the Bohemian regions of the Czech Republic. We do not have any information about the real origin of these families. The two BRCA2 mutations (c.8138 8142delCCTTT and c.8765 8766delAG) were found very frequently (41.5\% of BRCA2 mutations) in the Brno tested families, but were not identified in any of the families tested at the Prague centre. Interestingly, the spectra of BRCA2 mutations found in the two series of patients are different. In Prague only mutations in exons 10 and 11 were found, whereas in Brno mutations were scattered between exons 3 and 25 . The differences are likely to be a result of the smaller number of families tested in the Prague centre or by true differences between the inhabitants of Central Bohemia and other parts of the Czech Republic.

The preventive follow-up care for BRCA1 and BRCA2 carriers is well organized at high-risk clinics located in the Brno and Prague Oncology Centres. The quality of preventive follow-up in other centres needs to be studied because many carriers are followed in smaller hospitals all over the country.

The pre-screening of unselected breast and ovarian cancer patients for the presence of the three most frequent BRCA1 mutations which account for a large proportion of all BRCA1 and BRCA2 gene alterations in high-risk breast or ovarian cancer families (46.9\% in Brno and $57.6 \%$ in Prague) is warranted based on the findings of this report.

\section{Acknowledgements}

The work is supported by the Internal Grant Agency of the Ministry of Health of the Czech Republic, Grant No. NR 8022-3, NR 8213-3 and No. NC
7527-3; by the grant of the Ministry of Health of the Czech Republic MZO 00209805; Grant and Research Project of the Ministry of Education MSM 0021620808

\section{References}

1. Foretova L, Machackova E, Navratilova M, Pavlu H, Hruba M, Lukesova M and Valik D. BRCA1 and BRCA2 mutations in women with familial or early-onset breast/ovarian cancer in the Czech Republic. Hum Mutat 2004; 23: 397-398.

2. Pohlreich P, Zikan M, Stribrna J, Kleibl Z, Janatova M, Kotlas J, Zidovska J, Novotny J, Petruzelka L, Szabo C and Matous B. High proportion of recurrent germline mutations in the BRCAl gene in breast and ovarian cancer patients from the Prague area. Breast Cancer Res 2005; 7: R728-736.

3. Machackova E, Damborsky J, Valik D and Foretova L. Novel germline BRCA1 and BRCA2 mutations in breast and breast/ovarian cancer families from the Czech Republic. Hum Mutat 2001; 18: 545

4. Bartonkova H, Foretova L, Helmichova E, Kalabova R, Kleibl Z, Konopasek B, Krutilkova V, Machackova E, Novotny J, Petrakova K, Petruzelka L, Plevova P, Pohlreich P, Rob L, Skovajsova M, Vesely $\mathrm{J}$ and Zaloudik J. Recommendations for care of patients with breast and ovarian cancer and healthy individuals with germline mutations in BRCA1 or BRCA2 gene. Clinical Oncology 2003; 16: 28-34. In Czech.

5. Claes K, Poppe B, Machackova E, Coene I, Foretova L, De Paepe $A$ and Messiaen L. Differentiating pathogenic mutations from polymorphic alterations in the splice sites of BRCA1 and BRCA2. Genes Chromosomes Cancer 2003; 37: 314-320. 